\title{
Entanglement entropies and fermion signs of critical metals
}

\author{
N. Kaplis, ${ }^{1}$ F. Krüger, ${ }^{2,3}$ and J. Zaanen ${ }^{1}$ \\ ${ }^{1}$ Instituut-Lorentz for Theoretical Physics, Leiden University, Niels Bohrweg 2, Leiden 2333 CA, Netherlands \\ ${ }^{2}$ London Centre for Nanotechnology, University College London, Gordon Street, London WC1H OAH, United Kingdom \\ ${ }^{3}$ ISIS Facility, Rutherford Appleton Laboratory, Chilton, Didcot, Oxon OX11 0QX, United Kingdom
}

(Received 9 May 2016; revised manuscript received 24 February 2017; published 3 April 2017)

\begin{abstract}
The fermion sign problem is often viewed as a sheer inconvenience that plagues numerical studies of strongly interacting electron systems. Only recently has it been suggested that fermion signs are fundamental for the universal behavior of critical metallic systems and crucially enhance their degree of quantum entanglement. In this work we explore potential connections between emergent scale invariance of fermion sign structures and scaling properties of bipartite entanglement entropies. Our analysis is based on a wave-function Ansatz, that incorporates collective, long-range backflow correlations into fermionic Slater determinants. Such wave functions mimic the collapse of a Fermi liquid at a quantum critical point. Their nodal surfaces, a representation of the fermion sign structure in many-particle configurations space, show fractal behavior up to a length scale $\xi$ that diverges at a critical backflow strength. We show that the Hausdorff dimension of the fractal nodal surface depends on $\xi$, the number of fermions and the exponent of the backflow. For the same wave functions we numerically calculate the second Rényi entanglement entropy $S_{2}$. Our results show a crossover from volume scaling, $S_{2} \sim \ell^{\theta}$ ( $\theta=2$ in $d=2$ dimensions), to the characteristic Fermi-liquid behavior $S_{2} \sim \ell \ln \ell$ on scales larger than $\xi$. We find that volume scaling of the entanglement entropy is a robust feature of critical backflow fermions, independent of the backflow exponent and hence the fractal dimension of the scale invariant sign structure.
\end{abstract}

DOI: 10.1103/PhysRevB.95.155102

\section{INTRODUCTION}

Bipartite entanglement entropies have attracted much attention as quantum information measures in the many-body context (see Ref. [1] for a recent review). The idea is to divide the system into two spatial regions, $A$ and $B$ (see Fig. 1), and to compute the reduced density matrix of subsystem $A$ by taking a partial trace on the full density matrix, $\hat{\rho}_{A}=\operatorname{Tr}_{B} \hat{\rho}$. One can then compute the von Neumann entanglement entropy associated with the reduced density matrix by $S_{v N}=-\operatorname{Tr}\left(\hat{\rho}_{A} \ln \hat{\rho}_{A}\right)$ or, alternatively, the $n$th Rényi entropy by $S_{n}=\operatorname{Tr}\left(\hat{\rho}_{A}^{n}\right) /(1-n)$. The Rényi and von Neumann entanglement entropies are related to each other by the "replica limit," $S_{v N}=\lim _{n \rightarrow 1} S_{n}$, and they constitute measures of how the states in $A$ are entangled with those in $B$.

In gapped systems, the entanglement entropy scales with the area of the interface between the subsystems, $S_{v N} \sim S_{n} \sim$ $\ell^{d-1}$, simply because correlations are short ranged. Perhaps surprisingly, this area law turns out to be generic to bosonic ground states in dimensions $d \geqslant 2$. Its robustness was first demonstrated in the context of free bosonic field theories [2-4]. More recently, it has been shown that Goldstone modes in ordered systems with broken continuous symmetry [5], topological order in gapped systems [6], and the scale invariance at bosonic quantum critical points [7] give rise only to subleading, additive corrections to the area law. Across quantum phase transitions, cusp singularities are found in the prefactor of the area law [8,9]. Hence, the area law does not a priori carry information regarding a given phase of matter $[1,10]$.

In sharp contrast to the celebrated area-law scaling in the quantum ground states, finite-energy-density eigenstates typically satisfy a volume-law scaling, $S_{v N} \sim S_{n} \sim \ell^{d}$ [11-14]. Recently, Grover and Fisher [15,16] presented considerations that appear to shed light on the origin of the gross differences between (near) ground states and highly excited states, considering states of the form $|\Phi\rangle=\sum_{i} A_{i} \mid$ config, $\left.i\right\rangle$, where $\mid$ config, $i\rangle$ are the configurations of the $N$-particle Hilbert space in a certain local basis. They showed that various generic wave functions with all $A_{i} \geqslant 0$ cannot exceed an area-law entanglement [16]. This is consistent with the area-law bound for bosonic ground states: as first realized by Feynman [17,18], many-body ground-state wave functions of bosons in the coordinate representation are nodeless, and it is therefore possible to find a local basis in which all the amplitudes $A_{i}$ can be chosen to be positive definite. In an earlier paper [15], Grover and Fisher consider states such that all $A_{i}$ have the same absolute value but random signs, with the effect that the entanglement entropy acquires a volume scaling. Such states should be representative for highly excited energy eigenstates, given that these will form a dense continuum while all states have to be orthogonal to each other, which can be accomplished only when sign changes are maximally dense. These findings suggest that the scaling of the entanglement entropies is not determined by entanglement in a general sense but instead by the sign structure carried by the entangled state.

It is perhaps a universal affair that the sign structures of highly excited states are of such complexity that they can be regarded as random for all practical purpose. However, upon descending to low energy there might be room for more structure. This becomes especially relevant dealing with the plethora of problems that are characterized by the (fermion) sign problem. The vacuum states of interacting fermions at a finite density or generic quantum spin problems are characterized by sign changes that cannot be transformed away. Systems suffering from such sign problems no longer map on probabilistic systems, and these are claimed to be, in general, noncomputable: the computation of the vacuum is a NP-hard problem [19]. This fundamental fact appears to 


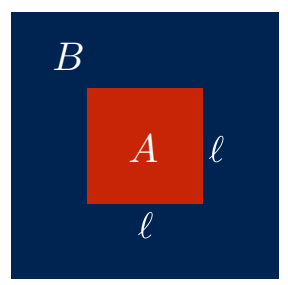

FIG. 1. Two-dimensional system divided into two subsystems, A and B. Subsystem B is traced out.

be overlooked in attempts to prove that in full generality the ground states associated with local Hamiltonians would be characterized by an area law for the entanglement entropy.

The only genuine sign-full vacuum that is under complete control is the ground state of the Fermi gas and its perturbative extension, the Fermi liquid. This is characterized by the antisymmetrization procedure explained in the quantum mechanics textbooks. This amounts to an irreducible long-range entanglement in a real-space representation involving only the exchange signs, which has proven to be very difficult to encode in, for instance, a tensor network, given its nonlocal nature. It is a famous result that its entanglement entropy scales like area-log-area, $S_{v N} \sim S_{n} \sim \ell^{d-1} \ln \ell$ [20-24], i.e., longer ranged than a typical bosonic system [25].

It is likely that non-Fermi-liquid states such as quantum critical metals are characterized by sign structures that are radically different from those of conventional metals, giving rise to a very different, universal form of entanglement scaling. This intuition is inspired by the strange metallic states that are predicted by the holographic duality [26], known as the Anti-de Sitter and conformal field theory (AdS-CFT) correspondence. These states appear to correspond generically to quantum critical phases characterized by an emergent scale invariance which does not require fine-tuning to critical points. It has been demonstrated [27] that such holographic strange metals can exhibit an anomalous entanglement-entropy scaling $S_{v N} \sim \ell^{\theta}$ with an exponent that can take any value $d-1<\theta \leqslant d$ and that is equal to the hyperscaling violation exponent [28]. While there is abundant evidence that the resulting vacua are "sign-full" non-Fermi liquids, their sign structures have not been investigated yet.

In the paper, we explore the connection between nontrivial sign structures and the scaling of bipartite entanglement entropies in the context of fermionic hydrodynamical backflow wave functions. It is not known whether these are eigenstates of any realistic Hamiltonian, but they have quite a history as a device to wire in a richer sign structure in numerical quantum Monte Carlo computations [29-33]. The highlight is that, in a certain parameter regime, backflow wave functions are characterized by a fractal distribution of zeros in configuration space [34]. This backflow system was therefore introduced as a model that might shed light on the nature of quantum critical non-Fermi-liquid states [34], revolving around the conjecture that a fractal nodal surface is a necessary condition for the emergence of scale invariance in any system characterized by "irreducible" signs in the ground state.

The outline of this paper is as follows. In Sec. II, we review the fermionic backflow wave-function Ansatz and introduce the surface of zeros of the wave function in configuration space as a geometrical measure of the sign structure. We illustrate the emergent scale invariance in the nodal structure and show that by generalizing the wave-function Ansatz used in previous work [34], it is possible to vary the fractal dimension of the nodal surface over a significant range. In Sec. III we numerically calculate the Rényi entanglement entropy $S_{2}$ for the states described by the generalized backflow wave functions in two dimensions. Our main finding is that irrespective of the fractal dimension of the nodal surface of the critical backflow state, the entanglement entropy follows a volume law. In Sec. IV, we summarize our results and discuss their implications.

\section{SIGN STRUCTURES OF BACKFLOW FERMIONS}

Fermion signs are expected [34] to play a crucial role for the universal behavior at quantum critical points. This insight is based on the constrained world-line path-integral reformulation [35] of the sign-full fermionic path integral, which is used in quantum Monte Carlo simulations to treat the fermion signs in a more manageable way [36-38]. In this language the nodes of the many particle density matrix impose hard-core constraints on the dynamics of effectively bosonic world lines. This nodal hypersurface can be viewed as a geometrical representation of the fermion sign structure in many-particle configuration space.

The Fermi energy $E_{F}$ is encoded in the constraint structure [34]: the average nodal pocket size gives rise to an average collision time $\tau_{c} \sim 1 / E_{F}$ of the world lines with the constraint structure. The observation of Planckian dissipation in the quantum critical region of high- $T_{c}$ cuprates [39], as well as the discontinuous Fermi-surface reconstruction [40] and the quasiparticle divergence [41] seen in heavy-fermion intermetallics, clearly shows that at the quantum critical point the metallic system loses its knowledge of the Fermi degeneracy scale. From the above considerations based on the constrained path integral it is therefore clear that the fermion sign structure has to become scale invariant.

It has been demonstrated [34] that collective, long-range backflow correlations built into fermionic wave functions can lead to emergent scale invariance in the nodal structure that goes hand in hand with a disappearance of the discontinuity in the single-particle momentum distribution $n(\boldsymbol{k})$. Fermionic backflow wave functions therefore provide a simple tool to study the collapse of a Fermi liquid at a critical point and to investigate how the Hausdorff dimension of the fractal nodal structure enters scaling relations. In this section we briefly review the properties of the fermionic backflow gas. We will then generalize the wave-function Ansatz used in previous work [34] and demonstrate that the fractal dimension can be tuned over a significant range, e.g., by changing the exponent of the long-range backflow correlations.

The idea to incorporate hydrodynamical backflow effects in quantum-mechanical wave functions dates back to Feynman and Cohen [42]. They argued that the "roton" in helium-4 is like a single mobile atom which is dressed by collective motions in the liquid. Helium is a nearly incompressible fluid, and the density in the neighborhood of the moving particle is barely altered. As a consequence, there has to be a backflow 
of other particles conserving the total current and leading to an enhancement of the effective mass of this quasiparticle. This can be described quantum mechanically by taking wave functions $\exp \left(i \boldsymbol{k} \cdot \tilde{\boldsymbol{r}}_{i}\right)$ with collective quasiparticle coordinates

$$
\tilde{\boldsymbol{r}}_{i}=\boldsymbol{r}_{i}+\sum_{j(\neq i)} \eta\left(\left|\boldsymbol{r}_{i}-\boldsymbol{r}_{j}\right|\right)\left(\boldsymbol{r}_{i}-\boldsymbol{r}_{j}\right)
$$

where $\boldsymbol{r}_{i}$ are the coordinates of the bare particles and $\eta(r)$ is a smoothly varying function which falls off like $\sim r^{-3}$ on large distances, exactly as the dipolar backflow in a classical, incompressible fluid. Much later, it was found that wave functions of the form $\Psi_{\mathrm{bf}}=J\left(\boldsymbol{r}_{1}, \ldots, \boldsymbol{r}_{N}\right) \operatorname{det}\left(e^{i \boldsymbol{k}_{i} \cdot \tilde{\boldsymbol{r}}_{j}}\right)$, with long-range back-flow correlations built into the Slater determinant, yield excellent variational Monte Carlo energies for bulk liquid ${ }^{3} \mathrm{He}$ [29], electron jellium [30,31], metallic hydrogen [32], and the two-dimensional Hubbard model [33]. Note that the Jastrow factors $J$ are positive definite and symmetric under particle exchange. Hence, the fermion sign structure is completely determined by the determinant factor and will crucially depend on the collective backflow. Following Ref. [34], we ignore the Jastrow factor (as well as the spin dependence) and consider backflow wave functions of the form

$$
\Psi\left(\boldsymbol{r}_{1}, \ldots, \boldsymbol{r}_{N}\right)=\mathcal{N} \operatorname{det}\left(e^{\imath \boldsymbol{k}_{i} \cdot \tilde{\boldsymbol{r}}_{j}}\right),
$$

where $\mathcal{N}$ denotes a normalization factor and the collective coordinates are defined in Eq. (1). We will use a generalized backflow function

$$
\eta(r)=a^{\beta} /\left(r^{\beta}+r_{0}^{\beta}\right),
$$

where $a$ determines the strength of the backflow and $r_{0}$ is a short-distance cutoff. The backflow exponent $\beta=3$ encodes for the literal hydrodynamical, dipolar backflow. We will also use different values of $\beta$ and show that this amounts to a flexible way to change the fractal dimension of the critical nodal surface. This represents the key result of this section. Over very small distances, the fractal behavior is cut off by $r_{0}$. All our results are independent of the particular choice of $r_{0}$, as long as it is chosen to be sufficiently small compared to the other length scales in the system.

The wave functions (2) are eigenstates of a freeparticle Hamiltonian in terms of the collective coordinates, $\mathcal{H}=-\frac{\hbar^{2}}{2 m} \sum_{i} \partial^{2} / \partial \tilde{\boldsymbol{r}}_{i}{ }^{2}$. In the following we will consider the ground state of this backflow gas for a two-dimensional square system of size $L \times L$ with periodic boundary conditions. This corresponds to a set of momenta $\boldsymbol{k}_{i}$ on a two-dimensional grid with spacing $\Delta k=2 \pi / L$ and $\left|\boldsymbol{k}_{i}\right| \leqslant k_{F}$; for example, for $k_{F}=2$ (in units of $\Delta k$ ) we obtain $N=13$ particles with momenta shown in the inset of Fig. 2(a).

The nodal hypersurface is determined by the zeros of the wave function, $\Psi\left(\boldsymbol{r}_{1}, \ldots, \boldsymbol{r}_{N}\right)=0$. The geometry and topology of this object characterize the sign structure of the fermionic state. In Fig. 2 we show two-dimensional cuts, which are obtained by fixing $N-1$ particles at random positions. For the noninteracting Fermi gas the nodal surface seen by the remaining particle smoothly connects the $N-1$ fixed particles, forming pockets with a typical size of the order of the interparticle spacing [Fig. 2(a)]. Note that for any fermionic wave function the $N-1$ particles are necessarily located on the nodal-surface cut because of Pauli's exclusion principle. (a)
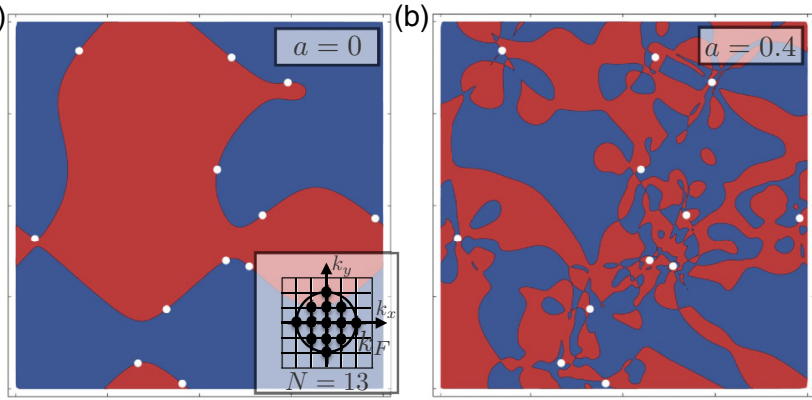

(c)
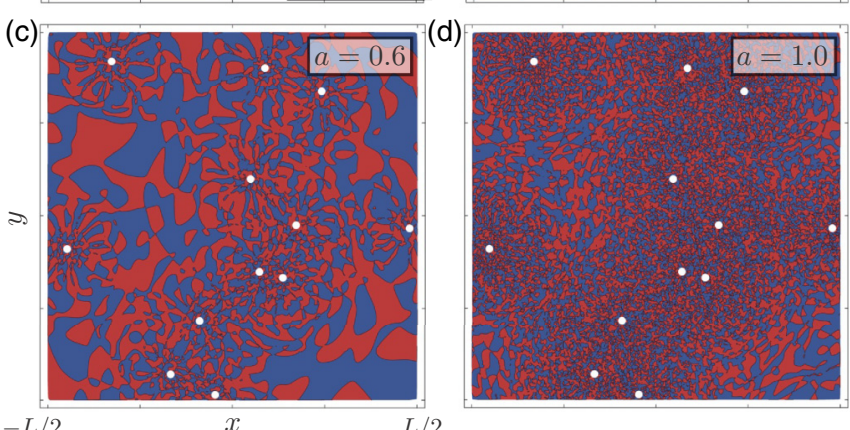

FIG. 2. Two-dimensional cuts of the nodal surface of fermionic backflow wave functions for $k_{F}=2(N=13)$, backflow exponent $\beta=3$, and increasing backflow strength $a$. The white dots show the fixed positions of $N-1$ particles. The nodal surface seen by the remaining particle is given by the interface between blue and red areas, corresponding to the two different signs of the wave function.

Figures 2(b)-2(d) show the evolution of the nodal surface as a function of the backflow strength $a$ for $\beta=3$.

In order to quantify the changes of the nodal surface and to demonstrate that backflow indeed leads to fractal behavior, we numerically calculate the correlation integral $C(r)$ which counts the number of pairs of nodal-surface points with separation less than $r$. For a fractal object, $C(r) \sim r^{\nu}$, with $v=$ $d_{H}$ being the Hausdorff or fractal dimension [34]. The resulting correlation integrals for $N=49, \beta=3$, and different values of the backflow strength $a$ are shown in Fig. 3. Without backflow, we find $v=1$ on scales smaller than the interparticle spacing $r_{s}$, consistent with smooth one-dimensional nodal lines. Near $r_{s}$ there exists a crossover to $v=2$, reflecting that the nodal structure looks two-dimensional on scales larger than the average spacing between nodal lines. For $a>0$ the correlation integrals show fractal behavior from the small-distance cutoff $r_{0}$ up to a "correlation-length" scale $\xi$, which increases with $a$ and seems to diverge at a critical value $a_{c} \approx 0.9-1.0$. At this value, the backflow strength $a$ is of the order of the interparticle spacing. Unfortunately, it is not possible to extract accurate values of $\xi$ and $a_{c}$ since the crossover is relatively broad for the system sizes we can study and since changes of $v$ become quite small near $a_{c}$. Interestingly, the fractal dimension $v$ is found to be nonuniversal and to increase with $a$ over quite a big range. This behavior is summarized in Fig. 4 for different particle numbers $N$ and backflow exponents $\beta$. As expected, the Hausdorff dimension depends on $\beta$. It also changes noticeably between $N=29$ and $N=49$, indicative of a relatively strong finite-size dependence. 

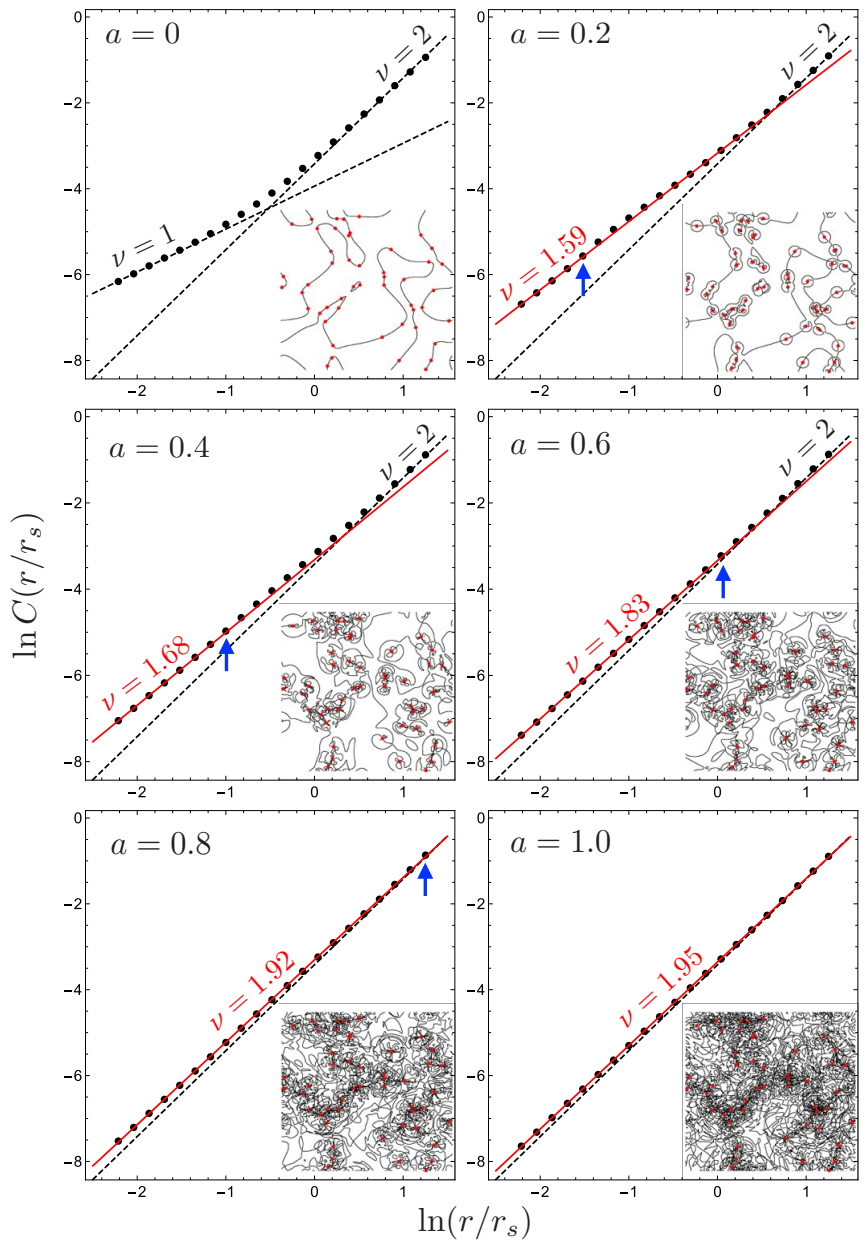

FIG. 3. Correlation integrals for $N=49, \beta=3$, and different values of the backflow strength $a$. Corresponding nodal-surface cuts are shown as insets. Both the range $\xi$ of fractal behavior (indicated by blue arrows) and the Hausdorff dimension $v$ increase with $a$. For $a=1.0, \xi$ exceeds the system size.

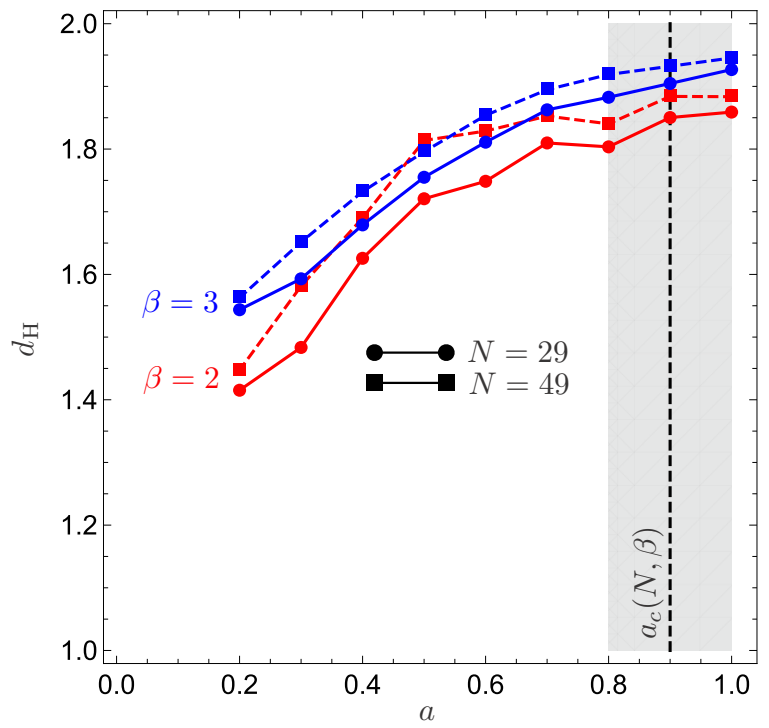

FIG. 4. Fractal dimension $d_{H}=v$ of the nodal surface as a function of the backflow strength $a$ for backflow exponents $\beta=2$ (red) and $\beta=3$ (blue) and different particle numbers $N$.
In our earlier work [34] we already observed that the Hausdorff dimension $d_{H}$ of the nodal surface does depend on $a$ but considered only the canonical value $\beta=3$ for the backflow exponent. Although the identification with the physical backflow notion is no longer possible, one can just exploit the freedom in the parametrization (3) as defining a family of wave-function Ansätze.

As it turns out, for a "longer-range" envelope function $(\beta<3), d_{H}$ decreases, while it increases for $\beta>3$. A typical example is shown in Fig. 4: one infers that relative to the $\beta=3$ results, the Hausdorff dimensions are significantly smaller for $\beta=2$. We will exploit the differences of these two cases in our study of the Rényi entropies. However, we have explored a larger family of envelope functions, finding that the Hausdorff dimension can, in principle, be tuned to have any value $1<d_{H}<2$.

\section{THE SECOND RÉNYI ENTROPY OF BACKFLOW FERMIONS}

Let us now turn to the results for the second Rényi entropy $S_{2}$ for the fermionic backflow wave functions (2), calculated numerically by using the Monte Carlo algorithm [43,44] outlined in the Appendix. The wave functions are defined on a square system of side length $L$ with periodic boundary conditions. We choose the subsystem $A$ to be an $\ell \times \ell$ square in the center of the system (see Fig. 1). We will compute $S_{2}(\ell / L)$ for different particle numbers $N$, for backflow strengths $a$, and for different values of the backflow exponent $\beta$. As we have shown in Sec. II, $\beta$ serves as a "knob" to change the Hausdorff dimension of the fractal nodal surface.

Before turning to the effects of backflow, let us first benchmark our code by calculating the entanglement entropy for free fermions $(a=0)$. We expect that $S_{2} \sim \ell \ln \ell$, at least in the regime $z:=\ell / L \ll 1$. For larger $z$, finite-size effects will start to become important. Since by construction the ground-state is nondegenerate, $S_{2}$ vanishes as $z \rightarrow 1$. At around $z=1 / \sqrt{2}$, where the area of subsystem $A$ is half of the total area of the system, the entanglement entropy

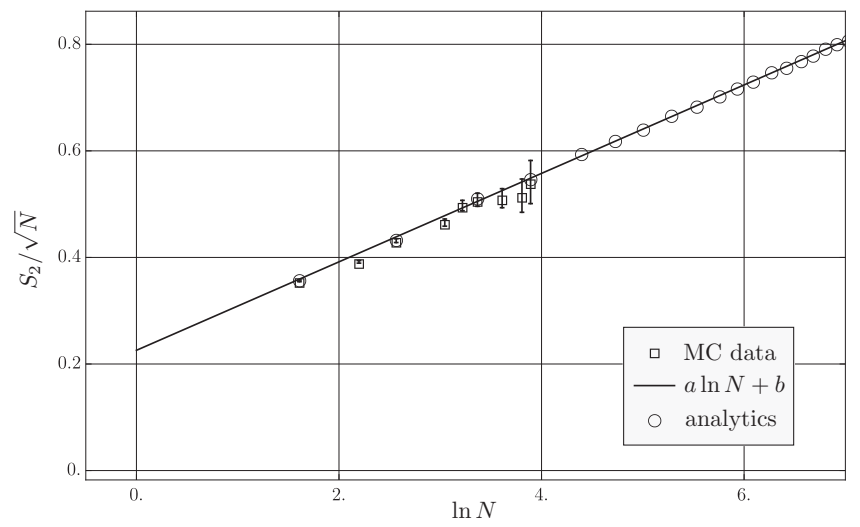

FIG. 5. Scaling of the second Rényi entropy vs the number of particles (or, equivalently, the size of the system) for the noninteracting Fermi gas $(a=0)$ and $z=0.3$. For comparison, we also show the analytic results obtained from the eigenvalues of the overlap matrix of single-particle states on the subvolume. 


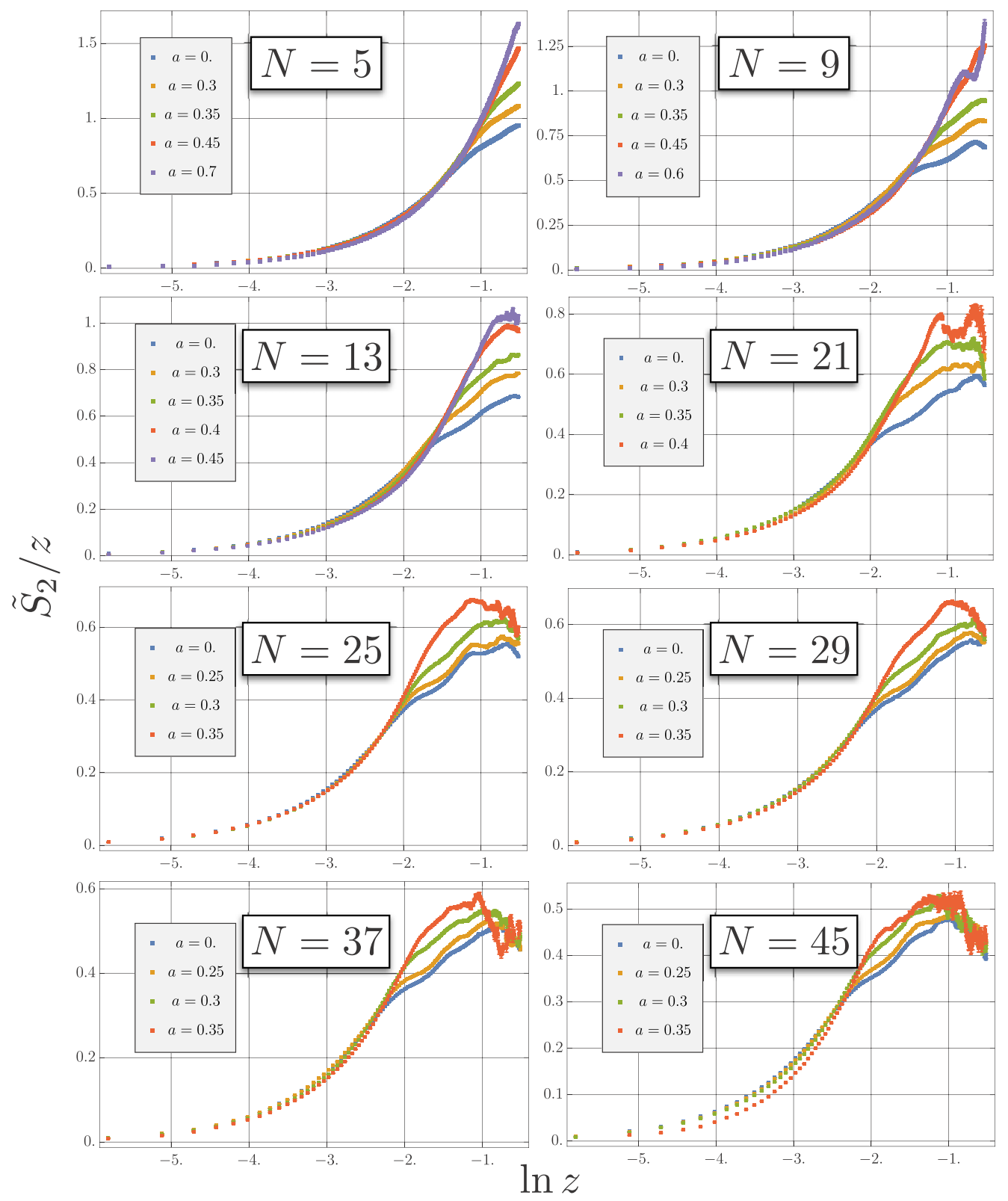

FIG. 6. Second Rényi entropy for the fermionic backflow system $(\beta=3)$ for increasing number of particles $N$ as a function of $z$. Increasing values of $a$ are overlaid. We have defined $\tilde{S}_{2}=S_{2} /(\sqrt{N} \ln N)$.

$S_{2}(z)$ has a maximum. In addition, commensuration effects between the partitioning and the periodic boundary conditions are known [45] to cause small ripples or oscillations in $S_{2}(z)$ that are most pronounced near the maximum of $S_{2}$. To minimize such finite-size effects, we will investigate entanglement entropies only for values $z<1 / 2$. We can look for the typical area-log-area Fermi-liquid scaling either as a function of $z$ or as a function of the particle number $N$. For fixed density $\rho=N / L^{2}$, one expects $S_{2} \sim \sqrt{N} \ln N$. However, it is known that for free fermions, the entanglement entropy contains a sizable subleading area-law contribution $\sim \sqrt{N}$. In Fig. 5 we demonstrate that our Monte Carlo (MC) data indeed follow the expected dependence $S_{2} / \sqrt{N}=$ $a \ln N+b$, with coefficients $a, b$ that depend on the particular value of $z$.
For comparison, we have also calculated the entanglement entropies $S_{2}$ of the free-fermion ground state analytically. This requires the computation of the eigenvalues $\lambda_{1}, \ldots, \lambda_{N}$ of the overlap matrix $A_{i j}=\int_{A} \phi_{i}^{*}(\boldsymbol{r}) \phi_{j}(\boldsymbol{r})$ between the single-particle states $\phi_{i}(\boldsymbol{r})=L^{-1} e^{i \boldsymbol{k}_{i} \cdot \boldsymbol{r}}$ on the subsystem $A$. The entanglement entropy is obtained as $S_{2}=-\sum_{i} \ln \left[\lambda_{i}^{2}+\left(1-\lambda_{i}\right)^{2}\right]$ [45]. As illustrated in Fig. 5, we find excellent quantitative agreement between the analytic results and out MC data.

We now look in detail at the effects of backflow, starting with a backflow exponent of $\beta=3$ and varying the backflow strength $a$. As demonstrated in Sec. II, backflow leads to fractal behavior in the nodal structure up to a length scale $\xi$. This length scale rapidly increases with $a$ and becomes of the order of the system size at $a_{c} \approx 0.9$, indicative of a 


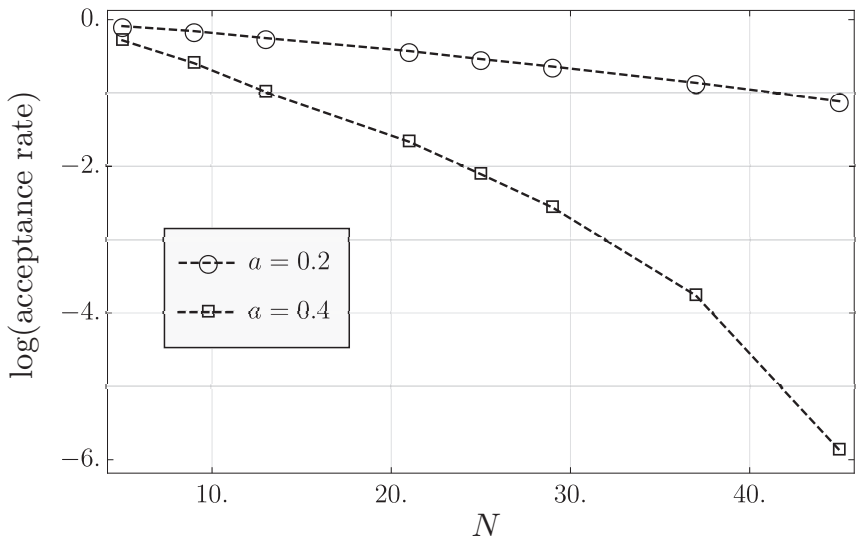

FIG. 7. The average acceptance rate for the Metropolis moves as a function of the number of particles for two values of the backflow strength, $a=0.2$ and $a=0.4$.

diverging correlation length. One would expect to see a change in the entanglement-entropy scaling from critical, non-Fermi-liquid behavior on scales $\ell<\xi$ to conventional Fermi-liquid behavior for $\ell>\xi$. As we will see below, this is indeed the case.

The results for the second Rényi entropy $S_{2}$ of the backflow system with given $N$ and $a$ as a function of $z=\ell / L$ are displayed in Fig. 6. A crossover in the entanglement-entropy scaling manifests itself as an inflection point in $S_{2}(z)$. The location of this inflection point shifts to larger values of $z$ as the backflow strength is increased, until our view is obscured by finite-size effects. Note that the inflection point in the case of the free Fermi gas is entirely due to finite-size effects and not indicative of a crossover in the entanglement scaling. As we will demonstrate later, for $a>0$ the entanglement entropy follows a volume law, $S_{2} \sim \ell^{2}$, on scales smaller than the length scale marked by the inflection point.

At this point it should be noted that we are able to calculate only the entanglement entropies for backflow systems of up to $N=45$ particles. Already for $N=49$ the noise increases significantly. For even larger systems the calculation becomes impossible. This restriction persisted even though our calculations were parallelized on the 'DutchGrid', an open platform for academic and research grid computing in the Netherlands, which allowed us almost unlimited CPU resources. What we observed was that the calculations become increasingly timeconsuming as the acceptance rate, for each move proposed in the Metropolis algorithm, gets exponentially suppressed as the number of particles increases. This is amplified for more strongly interacting systems, i.e., for larger values of the backflow strength $a$ (see Fig. 7). This is reminiscent of the critical slowing down observed in other quantum Monte Carlo simulations upon approaching a critical state.

Given the restricted number of particles available, one naturally worries about the reliability of our results against finite-size artifacts. After all, in bosonic systems one usually spans several orders of magnitude of system sizes in order to establish the thermodynamic limit. To address this issue and to demonstrate the amazing resilience of the entanglemententropy scaling of the fermionic backflow system to finite-size effects we study the dependence of our results on $N$. For this

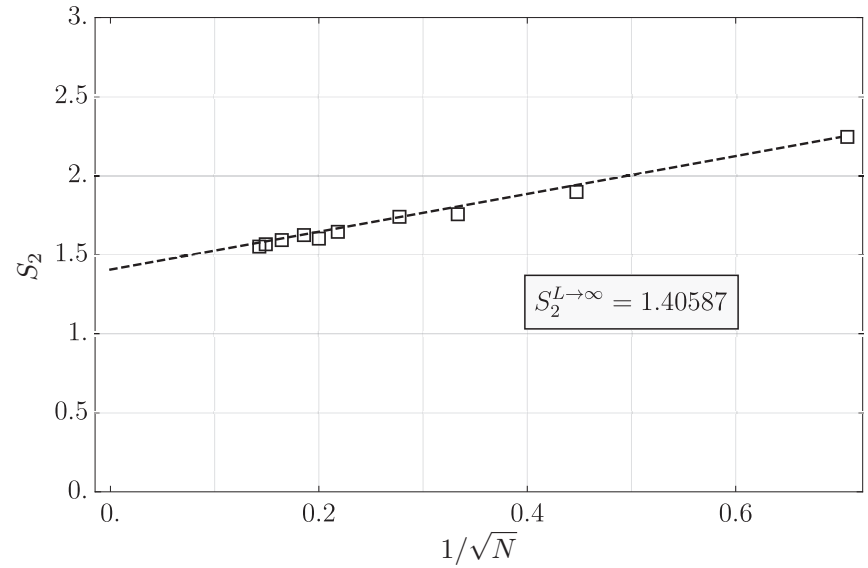

FIG. 8. The dependence of $S_{2}$ on the size of the system, keeping the density and the subsystem size constant. The entanglement entropies calculated for systems with a small number of particles are surprisingly close to the value for an infinite system.

purpose we fix the density $\rho=N / L^{2}$ of the system as well as the size $\ell$ of the subsystem and vary the total size of the system. In this way we can isolate the effect of the size of the total system and examine its effects on the entropy. More importantly, it means that the comparison of $S_{2}$ for different $N$, done this way, is meaningful.

It should be noted here that as the number of particles or the system size increases, the critical backflow strength $a_{c}$ decreases, resulting in noisier $S_{2}(z)$ curves. The fortunate caveat, however, is that as $N$ grows while $\rho$ and $\ell$ are kept fixed, $z$ becomes smaller. As can be seen in Fig. 6, the lower values of $z$ remain relatively reliable even for larger $N$. This is what gives us confidence in the scaling of $S_{2}$ vs $N$, presented in Fig. 8. It is quite remarkable that the convergence rate to the thermodynamic limit is very fast and that even in very small systems, e.g., for $N=5$ particles, one seems to be able to capture the essential physical behavior. Unfortunately, even for only $N=2$ fermions with backflow correlations, an analytic calculation of $S_{2}$ appears to be impossible.

We can now proceed to our ultimate goal, which is to compute the scaling behavior $S_{2} \sim \ell^{\theta}$ of the Rényi entanglement entropy of a critical backflow gas, $a \rightarrow a_{c}$. This can

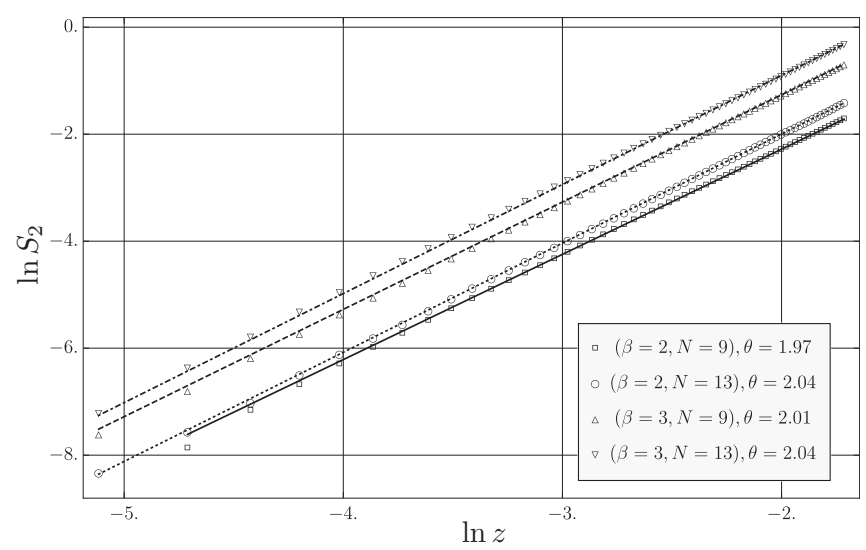

FIG. 9. Power-law behavior of the entanglement entropy, $S_{2} \sim \ell^{\theta}$, for different values of $\beta$ and $N$ and $a \rightarrow a_{c}$. 


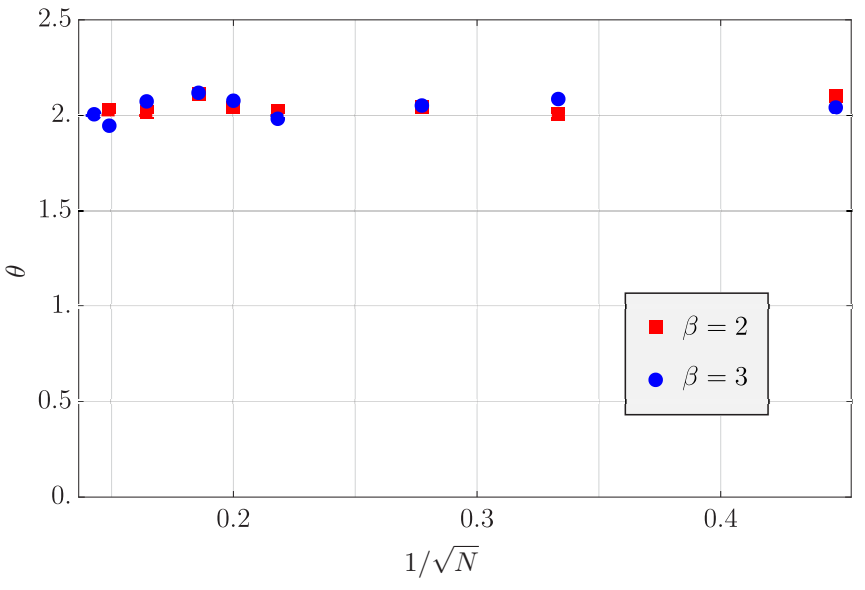

FIG. 10. The scaling exponent $\theta$ of the Rényi entropy as a function of the system size and for different values of the backflow exponent $\beta$.

be done by repeating the above scaling analysis for different values of $\ell$. We find very good evidence that for $a<a_{c}$ and sufficiently small $z$, the Rényi entropy always follows a volume law, $\theta=2$, irrespective of the value of $a$. This is in stark contrast to the behavior of the fractal dimension $d_{H}$ of the nodal surface, which increases with $a$ over a relatively large range (see Fig. 4).

In Fig. 9, we show the power-law behavior $S_{2} \sim \ell^{\theta}$ for different particle numbers $(N=9$ and $N=13)$ and backflow exponents $(\beta=2$ and $\beta=3)$. We find that the power laws are robust over a significant range with exponents that are very close to volume scaling, $\theta=2$. The deviations from a power law at very small scales are due to the small-distance cutoff $r_{0}$ of the backflow, Eq. (3). In Fig. 10 the extracted entanglement scaling exponents $\theta$ are shown for various particle numbers $N$. Our results show that $\theta$ is independent of $N$ and equal to $\theta=2$ within the error bars. Finally, we can change the backflow exponent from $\beta=3$ to $\beta=2$, which gives rise to a significant change in $d_{H}$ but does not affect the volume scaling of $S_{2}$.

\section{DISCUSSION AND CONCLUSIONS}

Let us reiterate our main findings. (i) The bipartite entanglement entropy is sensitive to the crossover between fractal and smooth behavior of the nodal structure. (ii) It is no possible to resolve the precise nature of the nontrivial fermion-sign structure in the fractal regime. We generically find here volume entanglement scaling, irrespective of the fractal dimension of the nodal structure.

The findings we have presented in this work do fit into a broader development. Although the bipartite entanglement entropies have played a stimulating role with regard to the introduction of quantum information notions in quantum many-body and field theory the realization is growing that it has severe shortcomings, to the degree that it may be plainly misleading. A first step has been the demonstration that it falls short of even detecting the quantum critical state of the transversal field Ising model in $2+1$ dimensions [10]. The work by Grover and Fisher [16] that formed the initial motivation of the present study is also devastating: the gross scaling properties of the bipartite entropies are insensitive to the infinite-party entanglement realized in configuration space, while the area law is just generic for a state characterized by a sign-free state where all wave-function amplitudes are positive definite. Given this observation, it appears to us that the widespread belief in the quantum information community that the ground states of systems described by any local Hamiltonian should show an area scaling of the bipartite entropy is actually based on folklore. Typically, the focus has been on ground states of systems that can be enumerated, like the (1+1)-dimensional systems and higher-dimensional incompressible spin systems which are invariably sign free. The (fermion) sign problem is just in the way of explicitly enumerating the ground states of sign-full systems, and these have been entirely ignored. These should exhibit a longer-range entanglement entropy, the simple case in point being the Fermi gas with its area-log-area scaling [46]. Given the sensitivity of the bipartite entanglement entropies to the presence of signs, the next question to ask is whether the bipartite entropies are sensitive to any special features in the sign structure. We set out to investigate that in this work. Given that basic scaling behavior is at stake, we perceive a sign structure that is organized as a fractal as an optimally beneficial circumstance for the Rényi entropy to reveal such specific sign information. The disappointing outcome is that the Rényi entropy appears to be capable of discriminating only between the Fermi gas (with the area-log-area scaling) and the denser sign structures characterized by fractal nodal surfaces. Although we did not check it explicitly, the odds are that, for a nodal surface with any Hausdorff dimension $d_{H}, S_{2}$ will exhibit a volume scaling.

A clear indication is the extremely rapid convergence of $S_{2}$ as a function of system size. As we emphasized in the previous section, the correct scaling behavior of the thermodynamic limit can already be obtained from a system containing as few as five fermions. This is in stark contrast to the geometry of the nodal surface itself, for which one needs at least 10 times as many particles to discern the fractal dimension, overcoming the finite-size artifacts. Obviously, this signals that the bipartite entanglement entropy, even in the presence of signs, is just revealing short-distance information. The precise mechanism is unclear to us.

Arguably, the only genuine non-Fermi liquids that have been identified based on controlled mathematics are the holographic strange metals. As we explained in the Introduction, their vacuum state may be characterized by bipartite von Neumann entropies with anomalous dimensions intermediate between area and volume scaling. However, this scaling dimension coincides with the hyperscaling violation exponent $\theta$ that is well established to characterize the deep infrared [26]. Given the message of the previous paragraph this appears to be an apparent paradox: how can it be that a quantity (the entanglement entropy) that appears to pick up only UV information in an explicit field-theoretical setting (the backflow fermions) manages to measure a deep IR scaling dimension (the $\theta$ of the holographic strange metals)? The caveat is, of course, that the first quantized backflow system, which is, in fact, based on a hidden free-fermion Ansatz, cannot be assigned a universal status that is representative of the sign structure of all quantum critical fermion systems. We leave the origin of the anomalous scaling of the entanglement entropy 
of the holographic strange metals as the great challenge for future research.

Given that the bipartite entanglement entropies are such blunt tools dealing with the complexities of sign-full quantum states of matter, are there alternatives? It seems obvious that sign structure and infinite-party, field-theoretical entanglement go hand in hand. This is generally recognized when dealing with highly excited states. This raises then the question of whether the nodal surface might serve the purpose of further characterizing the nature of this entanglement.

It has the obvious disadvantage that it requires too much information. In principle the full density matrix contains all quantum information of the state under consideration, but it is just too complex. The nodal surface is defined as the hypersurface of zeros of this density matrix, amounting to only a rather marginal improvement with regard to the information overload. However, the advantage of the nodal surface is that the sign structure is geometrized. A case in point is the very notion that the nodal surface can be characterized by either a smooth or a fractal geometry. In fact, because of the difficulty that one needs the state explicitly in order to enumerate the nodal surface, very little is known regarding this nodal surface geometry. The next difficulty is that the relationship between the nodal surface and the infinite-party entanglement is far from straightforward. Although not quite enumerated, the quantum nonlocality associated with the antisymmetrized states in the Fock space of the free-fermion gas has a mirror image in the nonlocality of the Fermi-gas nodal surface. Changing the position of one particle will change the precise locus of the nodal surface everywhere else. Obviously, turning the smooth nodal surface geometry into the fractal one of the backflow system, this degree of nonlocality is further enhanced, and one could then argue that the quantum critical fermion system is more densely entangled.

However, the lesson of the Bell pairs is that entanglement should be representation independent. Although it involves a nonlocal and highly singular transformation, the backflow system can be represented as a free-fermion gas of "backflow particles," and the denser entanglement associated with the bare-particle coordinates can therefore be viewed as a peculiarity of an inconvenient representation. Even for the Fermi gas itself there is a confusing issue with the precise status of the "antisymmetrization entanglement." The permutation signs surely block the way to a short-range entangled product state in real space. However, real space is a choice of representation. How does this work in single-particle momentum space? In fact, using the constrained path integral, one can prove easily that the Fermi gas precisely maps to the problem of a classical Mott insulator living in a harmonic well in momentum space [47]. Although this "Mottness" involves nonlocal information, this is the same kind of nonlocality perceived by a car stuck in a traffic jam: this has no relation whatever to the quantum information that may be used to factorize primes in polynomial time.

It is very clear that the fermion sign problem is coincident with the present incapacity of the available computational tools to deal with infinite-party long-range entangled states of thermodynamically large systems. But this incapacity does not necessarily imply the end of physics: especially, dealing with the incomputable sign-full vacuum states, there should be physics which may quite well be beautiful physics, as suggested by the holographic strange metals. An uncharted territory of physics is waiting behind the fermion sign brick wall, and we hope that our investigations will stimulate others to have a closer look.

\section{ACKNOWLEDGMENTS}

This work was carried out on the Dutch national einfrastructure with the support of the SURF Foundation. We are thankful to T. Grover, who suggested we have a look at the Rényi entropy of the backflow system and pointed out the algorithm explained in the Appendix. We acknowledge support of a grant from the John Templeton Foundation. The opinions expressed in this publication are those of the authors and do not necessarily reflect the views of the John Templeton Foundation.

\section{APPENDIX: COMPUTING THE RÉNYI ENTROPY: THE ALGORITHM}

In this appendix we review the Monte Carlo algorithm $[43,44]$ to calculate the second Rényi entropy $S_{2}$ for any given normalized $N$-particle wave function $\Psi\left(\boldsymbol{r}_{1}, \ldots, \boldsymbol{r}_{N}\right)$. This algorithm is used in Sec. III to calculate $S_{2}$ for the fermionic backflow wave functions (2). Notice that we expect $S_{v N}$ and $S_{n}$ to have the same scaling properties. $S_{2}$ is chosen because it is relatively easy to calculate.

In order to compute the bipartite entanglement entropy one needs to split the system into two subsystems, $A$ and $B$, as illustrated in Fig. 1. For brevity, we write the wave function as $\Psi\left(\boldsymbol{r}_{1}, \ldots, \boldsymbol{r}_{N}\right)=\Psi(\alpha, \beta)$, where $\alpha$ and $\beta$ are the configurations of subsystems $A$ and $B$, respectively (e.g., $\alpha$ is determined by the number $N_{A}$ of particles in subsystem $A$ and by the positions $\boldsymbol{r}_{1}, \ldots, \boldsymbol{r}_{N_{A}}$ of these particles and $N=N_{A}+N_{B}$ ). The reduced density matrix can then be written as

$$
\rho_{A}\left(\alpha, \alpha^{\prime}\right)=\sum_{\beta} \Psi^{*}(\alpha, \beta) \Psi\left(\alpha^{\prime}, \beta\right),
$$

where the partial trace over subsystem $B$ implies that $N_{A}=$ $N_{A}^{\prime}$. This, however, does not fix $N_{A}$ to be a particular number. One should view the above definition as a shorthand notation for

$$
\begin{aligned}
\rho_{A}\left(\alpha, \alpha^{\prime}\right)= & \delta_{N_{A}, 0}+\delta_{N_{A}, 1} \rho_{A}^{(1)}\left(\boldsymbol{r}_{1} ; \boldsymbol{r}_{1}^{\prime}\right)+\ldots \\
& +\delta_{N_{A}, N} \rho_{A}^{(N)}\left(\boldsymbol{r}_{1}, \ldots \boldsymbol{r}_{N} ; \boldsymbol{r}_{1}^{\prime}, \ldots, \boldsymbol{r}_{N}^{\prime}\right) .
\end{aligned}
$$

The exponentiated second Rényi entanglement entropy $e^{-S_{2}}=$ $\operatorname{Tr} \hat{\rho}_{A}^{2}$ can then be expressed as

$$
e^{-S_{2}}=\sum_{\alpha \alpha^{\prime} \beta \beta^{\prime}} \Psi^{*}(\alpha, \beta) \Psi\left(\alpha^{\prime}, \beta\right) \Psi^{*}\left(\alpha^{\prime}, \beta^{\prime}\right) \Psi\left(\alpha, \beta^{\prime}\right) .
$$

This expression has a simple physical interpretation. It is equal to the expectation value $e^{-S_{2}}=\left\langle\operatorname{Swap}_{B}\right\rangle$ of a SWAP operator that exchanges the configurations of subsystem $B$ between two replicas $\mathcal{S}, \mathcal{S}^{\prime}$ of the system, $\operatorname{SWAP}_{B}|\alpha, \beta\rangle\left|\alpha^{\prime}, \beta^{\prime}\right\rangle=$ $\left|\alpha, \beta^{\prime}\right\rangle\left|\alpha^{\prime}, \beta\right\rangle$.

Note that the sum in Eq. (A3) is equivalent to an integral over the positions $\boldsymbol{r}_{1}, \ldots, \boldsymbol{r}_{N}$ in $\mathcal{S}$ and $\boldsymbol{r}_{1}^{\prime}, \ldots, \boldsymbol{r}_{N}^{\prime}$ in $\mathcal{S}^{\prime}$. 
We will calculate this high-dimensional integral numerically, using Metropolis Monte Carlo integration. We will sample configurations using the probability distribution

$$
\begin{aligned}
P\left(\alpha, \beta ; \alpha^{\prime}, \beta^{\prime}\right) & =|\Psi(\alpha, \beta)|^{2}\left|\Psi\left(\alpha^{\prime}, \beta^{\prime}\right)\right|^{2} \\
& =\left|\Psi\left(\boldsymbol{r}_{1}, \ldots \boldsymbol{r}_{N}\right)\right|^{2}\left|\Psi\left(\boldsymbol{r}_{1}^{\prime}, \ldots, \boldsymbol{r}_{N}^{\prime}\right)\right|^{2}
\end{aligned}
$$

After a simple rewriting of the integrand in Eq. (A3), we find that we need to average

$$
F\left(\alpha, \beta ; \alpha^{\prime}, \beta^{\prime}\right)=\frac{\Psi\left(\alpha^{\prime}, \beta\right) \Psi\left(\alpha, \beta^{\prime}\right)}{\Psi(\alpha, \beta) \Psi\left(\alpha^{\prime}, \beta^{\prime}\right)}
$$

over Markov chains generated with the probability distribution $P$.
[1] N. Laflorencie, Phys. Rep. 646, 1 (2016).

[2] L. Bombelli, R. K. Koul, J. Lee, and R. D. Sorkin, Phys. Rev. D 34, 373 (1986).

[3] M. Srednicki, Phys. Rev. Lett. 71, 666 (1993).

[4] C. Callan and F. Wilczek, Phys. Lett. B 333, 55 (1994).

[5] A. B. Kallin, M. B. Hastings, R. G. Melko, and R. R. P. Singh, Phys. Rev. B 84, 165134 (2011).

[6] S. Furukawa and G. Misguich, Phys. Rev. B 75, 214407 (2007).

[7] M. A. Metlitski, C. A. Fuertes, and S. Sachdev, Phys. Rev. B 80, 115122 (2009).

[8] J. Helmes and S. Wessel, Phys. Rev. B 89, 245120 (2014).

[9] I. Frérot and T. Roscilde, Phys. Rev. Lett. 116, 190401 (2016).

[10] A. Chandran, V. Khemani, and S. L. Sondhi, Phys. Rev. Lett. 113, 060501 (2014).

[11] D. N. Page, Phys. Rev. Lett. 71, 1291 (1993).

[12] S. K. Foong and S. Kanno, Phys. Rev. Lett. 72, 1148 (1994).

[13] J. Sanchez-Ruiz, Phys. Rev. E 52, 5653 (1995).

[14] S. Sen, Phys. Rev. Lett. 77, 1 (1996).

[15] T. Grover and M. P. A. Fisher, J. Stat. Mech. (2014) P10010.

[16] T. Grover and M. P. A. Fisher, Phys. Rev. A 92, 042308 (2015).

[17] R. P. Feynman, Statistical Mechanics: A Set of Lectures (Addison-Wesley, Boston, 1972).

[18] C. Wu, Mod. Phys. Lett. B 23, 1 (2009).

[19] M. Troyer and U.-J. Wiese, Phys. Rev. Lett. 94, 170201 (2005).

[20] M. M. Wolf, Phys. Rev. Lett. 96, 010404 (2006).

[21] D. Gioev and I. Klich, Phys. Rev. Lett. 96, 100503 (2006).

[22] W. Li, L. Ding, R. Yu, T. Roscilde, and S. Haas, Phys. Rev. B 74, 073103 (2006).

[23] T. Barthel, M.-C. Chung, and U. Schollwöck, Phys. Rev. A 74, 022329 (2006).

[24] J. McMinis and N. M. Tubman, Phys. Rev. B 87, 081108 (2013).

[25] This behavior can yet be understood in a bosonic setting, albeit the corresponding boson problem is pathological. The area-logarea behavior is exhibited by any system carrying a spectrum of excitations characterized by a surface of dimension $d-1$ in single-particle momentum space of massless excitations which are governed at any point of this surface by a scale-invariant conformal field theory in $1+1$ dimensions. This is just an alternative way of naming a Fermi surface $[48,49]$, while also a highly fine-tuned frustrated Bose system can be tailored so that it is characterized by a "Bose surface" of gapless excitations at its quantum critical point [50]. For such "Bose metals" a logarithmic enhancement of the area law was found [51,52]. Self-evidently, gapless quantum spin liquids with a spinor Fermi surface also show a logarithmic correction to the area law [43].

[26] J. Zaanen, Y. Liu, Y. Sun, and K. Schalm, Holographic Duality in Condensed Matter Physics (Cambridge University Press, Cambridge, 2015).
[27] L. Huijse, S. Sachdev, and B. Swingle, Phys. Rev. B 85, 035121 (2012).

[28] D. S. Fisher, Phys. Rev. Lett. 56, 416 (1986).

[29] K. E. Schmidt, M. A. Lee, M. H. Kalos, and G. V. Chester, Phys. Rev. Lett. 47, 807 (1981).

[30] Y. Kwon, D. M. Ceperley, and R. M. Martin, Phys. Rev. B 48, 12037 (1993).

[31] Y. Kwon, D. M. Ceperley, and R. M. Martin, Phys. Rev. B 58, 6800 (1998).

[32] M. Holzmann, D. M. Ceperley, C. Pierleoni, and K. Esler, Phys. Rev. E 68, 046707 (2003).

[33] L. F. Tocchio, F. Becca, A. Parola, and S. Sorella, Phys. Rev. B 78, 041101 (2008).

[34] F. Krüger and J. Zaanen, Phys. Rev. B 78, 035104 (2008).

[35] D. M. Ceperley, J. Stat. Phys. 63, 1237 (1991).

[36] D. M. Ceperley, Phys. Rev. Lett. 69, 331 (1992).

[37] C. Pierleoni, D. M. Ceperley, B. Bernu, and W. R. Magro, Phys. Rev. Lett. 73, 2145 (1994).

[38] W. R. Magro, D. M. Ceperley, C. Pierleoni, and B. Bernu, Phys. Rev. Lett. 76, 1240 (1996).

[39] D. van der Marel, H. J. A. Molegraaf, J. Zaanen, Z. Nussinov, F. Carbone, A. Damascelli, H. Eisaki, M. Greven, P. H. Kes, and M. Li, Nature (London) 425, 271 (2003).

[40] S. Paschen, T. Lühmann, S. Wirth, P. Gegenwart, O. Trovarelli, C. Geibel, F. Steglich, P. Coleman, and Q. Si, Nature (London) 432, 881 (2004).

[41] J. Custers, P. Gegenwart, H. Wilhelm, K. Neumaier, Y. Tokiwa, O. Trovarelli, C. Geibel, F. Steglich, C. Pepin, and P. Coleman, Nature (London) 424, 524 (2003).

[42] R. P. Feynman and M. Cohen, Phys. Rev. 102, 1189 (1956).

[43] Y. Zhang, T. Grover, and A. Vishwanath, Phys. Rev. Lett. 107, 067202 (2011).

[44] M. B. Hastings, I. Gonzalez, A. B. Kallin, and R. G. Melko, Phys. Rev. Lett. 104, 157201 (2010).

[45] P. Calabrese, M. Mintchev, and E. Vicari, Europhys. Lett. 97, 20009 (2012).

[46] The same scaling has been found for a Fermi gas at unitarity with attractive interactions in the $s$-wave channel [W. J. Porter and J. E. Drut, Phys. Rev. B 94, 165112 (2016)].

[47] J. Zaanen, F. Krüger, J. H. She, D. Sadri, and S. I. Mukhin, Iran J. Phys. 8, 39 (2008).

[48] B. Swingle, Phys. Rev. Lett. 105, 050502 (2010).

[49] B. Swingle, Phys. Rev. B 86, 035116 (2012).

[50] A. Paramekanti, L. Balents, and M. P. A. Fisher, Phys. Rev. B 66, 054526 (2002).

[51] H.-H. Lai, K. Yang, and N. E. Bonesteel, Phys. Rev. Lett. 111, 210402 (2013)

[52] H.-H. Lai and K. Yang, Phys. Rev. B 93, 121109 (2016). 